\title{
Analytical and Numerical Study of Internal Representations in Multilayer Neural Networks with Binary Weights
}

\author{
Simona Coccol, Rémi Monassont and Riccardo Zecchina团 \\ 母 INFN and Dipartimento di Fisica, P.le Aldo Moro 2, I-00185 Roma, Italy \\ 1 Laboratoire de Physique Théorique de l'ENS, 24 rue Lhomond, 75231 Paris cedex 05, France \\ \# INFN and Dipartimento di Fisica, Politecnico di Torino, C.so Duca degli Abruzzi 24, I-10129 \\ Torino, Italy
}

\begin{abstract}
We study the weight space structure of the parity machine with binary weights by deriving the distribution of volumes associated to the internal representations of the learning examples. The learning behaviour and the symmetry breaking transition are analyzed and the results are found to be in very good agreement with extended numerical simulations.
\end{abstract}

PACS Numbers : 05.20 - $64.60-87.10$

Typeset using REVTEX 


\section{INTRODUCTION}

The understanding of the learning process of neural networks is of great importance from both theoretical and applications points of view [1]. While the properties of the simplest neural network, the perceptron, are now well explained, the picture we have for the learning phase of the far more relevant case of multilayer neural networks remains unsatisfactory. Due to the internal degrees of freedom present in multilayer networks (the state variables of the hidden units), the structure of the weight space inherited from the learning procedure is highly non trivial [1, 2, 3, 3, 4, 5, , 7].

Gardner's framework of statistical mechanics [3] has been proven to be useful in understanding the learning process by providing some bounds on the optimal performances of neural networks. In particular, it has allowed to derive the storage capacity and the generalization abilities of neural networks inferring a rule by example. However, the drawback of such an approach is that it does not give any microscopic information concerning the internal structure of the coupling space, in particular about internal representations.

Recently, an extension of Gardner's approach has been proposed [2] which leads to a deeper insight on the structure of the weight space by looking at the components of the latter corresponding to different states of the internal layers of the network. Such an approach has been successful in explaining some known features of multilayer neural networks and has permitted to find some new results concerning their learning-generalization performances as well as to make a rigorous connection with information theory [2, 11].

In this paper we focus on multilayer neural networks with binary weights [0]. This allows us to compare the analytical study with extensive numerical simulations and thus to provide a concrete check of the liability of the theory. Indeed, both the structure of internal representations and the (symmetry-breaking) learning phase transition predicted by our theory turn out to be in remarkable agreement with the numerical findings.

The paper is organized as follows. In section 2, we present our method from a general point of view and apply it to the parity machine with binary weights in Section 3. Section 
4 is devoted to numerical simulations. Our results are summed up in the conclusion.

\section{DISTRIBUTION OF THE INTERNAL REPRESENTATION VOLUMES}

As discussed in ref. [2], the method we adopt consists in a rather natural generalization of the well known Gardner approach based on the study of the fractional weight space volume not ruled out by the optimal, yet unknown, learning process [3]. We analyze the detailed decomposition of such volume in elementary volumes each one associated to a possible internal representations of the learned examples. The dynamical variables entering the statistical mechanics formalism are the (binary valued) interaction couplings and the spin-like states of the hidden units.

In what follows, we focus on non-overlapping multilayer networks composed of $K$ perceptrons with weights $J_{\ell i}$ and connected to $K$ sets of independent inputs $\xi_{\ell i}(\ell=1, \ldots, K$, $i=1, \ldots, N / K)$.

The learning process may be thought of as a two step geometrical process taking place in the weight space from the input to the hidden layer. First the $N / K$-dimensional subspace belonging to the $\ell$-th perceptron (or hidden unit) is divided in a number of volumes $\left(\leq 2^{P}\right)$, each of which being labeled by a $P$-components vector

$$
\tau_{\ell}^{\mu}=\operatorname{sign}\left(\vec{J}_{\ell} \vec{\xi}_{\ell}^{\mu}\right), \quad \ell=1, \ldots, K, \quad \mu=1, \ldots, P
$$

$\tau_{\ell}^{\mu}$ is the spin variable representing the state of the $\ell-$ th hidden unit when pattern number $\mu$ is presented at the input. Next, the solution space is defined as the direct product of the volumes belonging to all hidden nodes and satisfying the condition imposed by the decoder function

$$
f\left(\left\{\tau_{\ell}^{\mu}\right\}\right)=\sigma^{\mu}
$$

where $\sigma^{\mu}$ is the output classifying the input pattern. The overall space of solution is thus composed by a set of internal volumes $V_{\mathcal{T}}$ identified by the $K \times P$ matrix $\tau_{\ell}^{\mu}$ called internal representation of the learning examples. The computation of the whole distribution of 
volumes $V_{\mathcal{T}}$, both their typical size and their typical number, yields a deeper understanding on the storage problem by the comparison of the number $\exp \left(\mathcal{N}_{D}\right)$ of volumes giving the dominant contribution to Gardner's volume with the upper bound given by total number $\exp \left(\mathcal{N}_{R}\right)$ of non-empty volumes (i.e. the total number of implementable internal representations). Moreover, the physics of the learning transition (the freezing phenomena and the replica symmetry breaking transition) acquires a detailed geometrical interpretation.

Here we consider the case of Parity Machines which are characterized by a decoder function defined as the product of the internal representation, $\sigma^{\mu}=f\left(\left\{\tau_{\ell}\right\}\right)=\prod_{\ell} \tau_{\ell}$.

As mentioned, given a set of $P=\alpha N$ binary input-output random relations, the learning process can be described as a geometrical selection process aimed to finding a suitable set of internal representations $\mathcal{T}=\left\{\tau_{\ell}^{\mu}\right\}$ characterized by a non zero elementary volume $V_{\mathcal{T}}$ defined by

$$
V_{\mathcal{T}}=\sum_{J_{\ell i}= \pm 1} \prod_{\mu} \theta\left(\sigma^{\mu} f\left(\left\{\tau_{\ell}^{\mu}\right\}\right)\right) \prod_{\mu, \ell} \theta\left(\tau_{\ell}^{\mu} \sum_{i} J_{\ell i} \xi_{\ell i}^{\mu}\right)
$$

where $\theta(\ldots)$ is the Heaviside function. The overall volume of the weight space available for learning (the Gardner volume $V_{G}$ ) can be written as

$$
V_{G}=\sum_{\mathcal{T}} V_{\mathcal{T}}
$$

For the learning problem, the distribution of volumes can be derived through the freeenergy

$$
g(r)=-\frac{1}{N r} \overline{\ln \left(\sum_{\mathcal{T}} V_{\mathcal{T}}^{r}\right)}
$$

by calculating the entropy $\mathcal{N}[w(r)]$ of the volumes $V_{\mathcal{T}}$ whose inverse sizes are equal to $w(r)=-\frac{1}{N} \ln V_{\mathcal{T}}$, given by the Legendre relations

$$
w(r)=\frac{\partial(r g(r))}{\partial r}, \quad \mathcal{N}[w(r)]=-\frac{\partial g(r)}{\partial(1 / r)} .
$$

When $N \rightarrow \infty, \frac{1}{N} \overline{\ln \left(V_{G}\right)}=-g(r=1)$ is dominated by volumes of size $w(r=1)$ whose corresponding entropy (i.e. the logarithm of their number divided by $N$ ) is $\mathcal{N}_{D}=$ 
$\mathcal{N}[w(r=1)]$ and, at the same time, the most numerous ones are those of smaller size $w(r=0)$ (since in the limit $r \rightarrow 0$ all the $\mathcal{T}$ are counted irrespective of their relative volumes) whose entropy $\mathcal{N}_{R}=\mathcal{N}[w(r=0)]$ is the (normalized) logarithm of the total number of implementable internal representations. Both $\mathcal{N}_{D}$ and $\mathcal{N}_{R}$ allow to built a rigorous link between statistical mechanics and information theory. The former $\left(\mathcal{N}_{D}\right)$ coincides with the

quantity of information $\mathcal{I}=-\sum_{\mathcal{T}} \frac{V_{\mathcal{T}}}{V_{G}} \log \frac{V_{\mathcal{T}}}{V_{G}}$ contained in the internal representation distribution $\mathcal{T}$ and concerning the weights whereas the latter $\left(\mathcal{N}_{R}\right)$ is the information capacity of the system, i.e. the maximal quantity information one can extract from the knowledge of the internal representations [2].

\section{ANALYTICAL CALCULATION FOR THE BINARY PARITY MACHINE}

In the following, we shall apply the above method to derive the weight space structure of the non-overlapping parity machine with binary couplings. The analysis of binary models [5] is indeed more complicated than that of their continuous counterpart due to Replica Symmetry Breaking (RSB) effects. However, in the binary case extensive numerical simulations on finite systems become available allowing for a very detailed check of the theory.

In the computation of $g(r), \mathcal{N}[w(r)]$ and $w(r)$ one assumes that, due to their extensive character, the self-averaging property holds. We proceed in the computation of the $g(r)$ following the scheme presented in [2,11] and discussed above. The basic technical difference with the standard Gardner approach resides in the double analytic continuation inherited from the presence of two sets of replica indices in the weight vectors. The first coming from the integer power $r$ of the internal volumes appearing in the partition function, the second from the replica trick.

The replicated partition function reads

$$
\left(\sum_{\left\{\tau_{\ell}^{\mu}\right\}} V_{\mathcal{T}}^{r}\right)^{n}=\sum_{\left\{\tau_{\ell}^{\mu \alpha}\right\}} \sum_{\left\{J_{\ell i}^{\alpha \nu}\right\}} \prod_{\alpha, \mu}\left[\prod_{\nu}\left(\prod_{\ell} \theta\left(\tau_{\ell}^{\mu \alpha} \sum_{i} J_{\ell i}^{\alpha \nu} \xi_{\ell i}^{\mu}\right)\right) \theta\left(\prod_{\ell} \tau_{\ell}^{\mu \alpha}\right)\right]
$$

with $\nu=1, \ldots, r$ and $\alpha=1, \ldots, n$ and which in turn implies the introduction of four 
sets of order parameters. In the above formula, with no loss of generality, we have posed $\sigma^{\mu}=1, \quad \forall \mu$.

At variance with Gardner's approach, the partition function (7) requires a double configuration trace, over the internal state variables and the binary couplings. We find

$$
g(r)=-\operatorname{Extr}_{Q_{\ell} \hat{Q}_{\ell}} \frac{1}{r} \mathcal{F}\left(Q_{\ell} \hat{Q}_{\ell}\right)
$$

where $\mathcal{F}$ reads

$$
\begin{gathered}
\mathcal{F}\left(Q_{\ell} \hat{Q}_{\ell}\right)=\frac{1}{2 K} \sum_{\ell} \operatorname{Tr}\left(Q_{\ell} \hat{Q}_{\ell}\right)+\frac{1}{K} \sum_{\ell} \ln \left[\operatorname{Tr}_{\left\{\vec{J}_{\ell}\right\}} e^{\frac{1}{2} \vec{J}_{\ell} \hat{Q}_{\ell} \vec{J}_{\ell}}\right]+ \\
+\alpha \ln \left[\operatorname{Tr}_{\left\{\tau_{\ell}^{\alpha}\right\}} \theta\left(\prod_{\ell} \tau_{\ell}^{\alpha}\right) \int \prod_{\ell} \frac{d \vec{x}_{\ell} d \overrightarrow{\hat{x}}_{\ell}}{2 \pi} \prod_{\alpha, \nu, \ell} \theta\left(x_{\ell}^{\alpha \nu} \tau_{\ell}^{\alpha}\right) e^{-\frac{1}{2}\left(\sum_{\ell} \overrightarrow{\hat{x}}_{\ell} Q_{\ell} \vec{x}_{\ell}+\sum_{\ell} \vec{x}_{\ell} \vec{x}_{\ell}\right)+i \sum_{\ell} \vec{x}_{\ell} \vec{x}_{\ell}}\right],
\end{gathered}
$$

with $\vec{x}_{\ell}, \overrightarrow{\hat{x}}_{\ell}, \vec{J}_{\ell}(n \times r)$-dimensional vectors. The elements of the $(n \times r) \times(n \times r)$ matrices $Q_{\ell}$ e $\hat{Q}_{\ell}$ are the overlaps

$$
q_{l}^{\alpha, \nu_{1}, \beta, \nu_{2}}=\frac{K}{N} \sum_{i} J_{\ell i}^{\alpha \nu_{1}} J_{\ell i}^{\beta \nu_{2}}
$$

between two coupling vectors belonging to the same hidden unit $\ell$ and their conjugate variables. The simplest non trivial Ansatz (which can be physically understood within the cavity approach [10]) on the structure of the above matrices, the Replica Symmetric (RS) Ansatz of our approach, must distinguish elements with $\alpha=\beta$ or $\alpha \neq \beta$, whereas ignores difference between replica blocks and between hidden units. The matrices $Q_{\ell}, \hat{Q}_{\ell}$ become independent of $\ell$ and with elements

$$
\begin{array}{ll}
q_{\ell}^{\alpha=\beta, \nu_{1}, \nu_{2}}=q^{*}, & \hat{q}_{\ell}^{\alpha=\beta, \nu_{1}, \nu_{2}}=\hat{q}^{*} \\
q_{\ell}^{\alpha \neq \beta, \nu_{1}, \nu_{2}}=q_{0}, & \hat{q}_{\ell}^{\alpha \neq \beta, \nu_{1}, \nu_{2}}=\hat{q}_{0}
\end{array}
$$

We then find

$$
\begin{aligned}
& g\left(r, \hat{q}_{0}, q_{0}, \hat{q}^{*}, q^{*}\right)=-\frac{1}{2} r q_{0} \hat{q}_{0}+\frac{1}{2}(r-1) q^{*} \hat{q}^{*}+\frac{1}{2} \hat{q}^{*}- \\
& \frac{1}{r} \int \Delta x \ln \int \Delta y\left(2 \cosh \left(\sqrt{\hat{q}_{0}} x+\sqrt{\hat{q}^{*}-\hat{q}_{0}} y\right)\right)^{r}- \\
& -\frac{\alpha}{r} \int \prod_{l} \Delta y_{l} \ln \left[\operatorname{Tr}_{\left\{\tau_{l}\right\}} \prod_{l=1}^{k} \int \Delta x_{l} H\left(\frac{\sqrt{\hat{q}^{*}-\hat{q}_{0}} x_{l}+\tau_{l} \sqrt{q_{0}} y_{l}}{\sqrt{1-\hat{q}^{*}}}\right)^{r}\right]
\end{aligned}
$$


where we have posed $\operatorname{Tr}_{\left\{\tau_{\ell}\right\}} \equiv \operatorname{Tr}_{\left\{\tau_{\ell}\right\}} \theta\left(\prod_{\ell} \tau_{\ell}\right), \Delta x=\exp \left(-x^{2} / 2\right) / \sqrt{2 \pi}$ and $H(y)=\int_{y}^{\infty} \Delta x$. One may notice that the above expression evaluated for $r=1$ reduces to the RS Gardner's like result on the parity machine [1] independent on the parameters $q^{*}$ and $\hat{q}^{*}$

$$
g\left(r=1, \hat{q}_{0}, q_{0}, \hat{q}^{*}, q^{*}\right)=G_{R S}\left(q_{0}, \hat{q}_{0}\right)=-\frac{1}{N} \overline{\ln V_{G}} .
$$

where $V_{G}$ is the Gardner volume. The geometrical organization of the domains is thus hidden in the Gardner volume and shows up only when $r \neq 1$ or if derivatives with respect to $r$ are considered, leading to an explicit dependence on the order parameters $q^{*}, \hat{q}^{*}$

$$
g\left(r=1+\varepsilon, \hat{q}_{0}, q_{0}, \hat{q}^{*}, q^{*}\right)=G_{R S}\left(q_{0}, \hat{q}_{0}\right)+\left.\varepsilon \frac{\partial g}{\partial r}\left(\hat{q}_{0}, q_{0}, \hat{q}^{*}, q^{*}\right)\right|_{r=1}
$$

In particular, the functions $\mathcal{N}[w(r=1)]$ and $w(r=1)$, being derivatives of $g(r)$, will depend on $q^{*}$ and $\hat{q}^{*}$.

The RS saddle point equations read:

1) $\frac{\partial g(r)}{\partial \hat{q}_{0}}=0$ :

$$
q_{0}=\int \Delta x \frac{\left[\int \Delta y\left(\cosh ^{r}\left(\sqrt{\hat{q}_{0}} x+\sqrt{\hat{q}^{*}-\hat{q}_{0}} y\right)\right) \tanh \left(\sqrt{\hat{q}_{0}} x+\sqrt{\hat{q}^{*}-\hat{q}_{0}} y\right]^{2}\right.}{\left[\int \Delta y \cosh ^{r}\left(\sqrt{\hat{q}_{0}} x+\sqrt{\hat{q}^{*}-\hat{q}_{0}} y\right)\right]^{2}},
$$

2) $\frac{\partial g(r)}{\partial \hat{q}^{*}}=0$ :

$$
q^{*}=\int \Delta x \frac{\int \Delta y \cosh ^{r}\left(\sqrt{\hat{q}_{0}} x+\sqrt{\hat{q}^{*}-\hat{q}_{0}} y\right) \tanh ^{2}\left(\sqrt{\hat{q}_{0}} x+\sqrt{\hat{q}^{*}-\hat{q}_{0}} y\right)}{\int \Delta y \cosh ^{r}\left(\sqrt{\hat{q}_{0}} x+\sqrt{\hat{q}^{*}-\hat{q}_{0}} y\right)},
$$

3) $\frac{\partial g(r)}{\partial q_{0}}=0$ :

$$
\hat{q}_{0}=\frac{\alpha K}{2 \pi\left(1-q^{*}\right)} \int \prod_{\ell} \Delta y_{\ell} \frac{\left[\operatorname{Tr}_{\left\{\tau_{\ell}\right\}} \tau_{1} \prod_{\ell=2}^{K} \int \Delta x_{\ell} H^{r}\left(A_{\ell}\right) \int \Delta x_{1} H^{r-1}\left(A_{1}\right) e^{-A_{1}^{2}}\right]^{2}}{\left[\operatorname{Tr}_{\left\{\tau_{\ell}\right\}} \prod_{\ell} \int \Delta x_{\ell} H^{r}\left(A_{\ell}\right)\right]^{2}}
$$

in which

$$
A_{\ell}=\frac{\sqrt{q^{*}-q_{0}} x_{\ell}+\sqrt{q_{0}} \tau_{\ell} y_{\ell}}{\sqrt{1-q^{*}}}
$$

4) $\frac{\partial g(r)}{\partial q^{*}}=0$ :

$$
\hat{q}^{*}=-\frac{\alpha K}{2 \pi\left(1-q^{*}\right)} \int \prod_{\ell} \Delta y_{\ell} \frac{\operatorname{Tr}_{\left\{\tau_{\ell}\right\}} \prod_{\ell=2}^{K}\left[\int \Delta x_{\ell} H^{r}\left(A_{\ell}\right)\right]}{\operatorname{Tr}_{\left\{\tau_{\ell}\right\}} \prod_{\ell} \int \Delta x_{\ell} H^{r}\left(A_{\ell}\right)} \int \Delta x_{1} H^{r-2}\left(A_{1}\right) e^{-A_{1}^{2}} .
$$


The case of the parity machine is relatively simple in that a consistent solution for the first two equations leads $q_{0}=0$ and $\hat{q}_{0}=0$ (as it happens in the computation of $V_{G}$ 迪.5]), which means that the domains remain uncorrelated during the learning process. The latter two equations simplify to

$$
\begin{gathered}
q^{*}=\frac{\int \Delta y \cosh ^{r}\left(\sqrt{\hat{q}^{*}} y\right) \tanh ^{2}\left(\sqrt{\hat{q}^{*}} y\right)}{\int \Delta y \cosh ^{r}\left(\sqrt{\hat{q}^{*}} y\right)}, \\
\hat{q}^{*}=\frac{\alpha K}{2 \pi\left(1-q^{*}\right)} \frac{\int \frac{d x}{\sqrt{2 \pi}} e^{-\frac{x^{2}\left(1+q^{*}\right)}{2\left(1-q^{*}\right)}} H^{r-2}\left(\sqrt{\frac{q^{*}}{1-q^{*}}} x\right)}{\int \Delta x H^{r}\left(\sqrt{\frac{q^{*}}{1-q^{*}}} x\right)},
\end{gathered}
$$

with a free energy given by

$$
\begin{aligned}
g\left(r, q^{*}, \hat{q}^{*}\right) & =-\frac{1}{2}(1-r) q^{*} \hat{q}^{*}+\frac{\hat{q}^{*}}{2}-\frac{1}{r} \ln \int \Delta y 2^{r} \cosh ^{r}\left(\sqrt{\hat{q}^{*}} y\right)+ \\
& -\frac{\alpha}{r}(K-1) \ln 2-\frac{\alpha}{r} K \ln \int \Delta x H^{r}\left(\sqrt{\frac{q^{*}}{1-q^{*}}} x\right) .
\end{aligned}
$$

For the parameters $q^{*}, \hat{q}^{*}$ there are two kinds of solution: a first one $q^{*}=1, \hat{q}^{*}=\infty$, which leads $w(r)=0$ and $\mathcal{N}[w(r)]=(1-a) \ln 2$ independently on $r$. The second kind must be computed numerically form (20) and (21).

In the replica theory, the choice of the right saddle solution, i.e. the maximization or the minimization of the free energy, is not completely straightforward due to the unusual $n \rightarrow 0$ analytic continuation [9]. Here we must deal with a double analytic continuation and the overall criterion that must be followed is given by

$$
\begin{aligned}
& r<0, \quad q_{0} \rightarrow \text { MAX, } q^{*} \rightarrow \text { MIN } \\
& 0<r<1, \quad q_{0} \rightarrow \text { MAX, } q^{*} \rightarrow \text { MAX } \\
& r>1, \quad q_{0} \rightarrow \text { MAX, } q^{*} \rightarrow \text { MIN },
\end{aligned}
$$

where $M A X$ or $M I N$ indicates whether one must chose the solution which maximizes or minimizes the free energy $g(r)$ respectively.

Like the zero entropy criterion for the binary perceptron, the behaviour of $\mathcal{N}[w(r)]$ and $w(r)$ (the cases $r=0$ and $r=1$ being of particular interest) tells us when the RS Ansatz 
breaks down. Notice that in the binary case also the volume size $w(r)$ assumes the role of an entropy in that it coincides with (minus) the logarithm of the normalized number of binary weight vectors belonging to a domain.

The Legendre transforms (6) of $g(r)$ lead to the formulas

$$
\begin{gathered}
w(r)=r q^{*} \hat{q}^{*}+\frac{1}{2} \hat{q}^{*}\left(1-q^{*}\right)-\frac{\int \Delta y \cosh ^{r}\left(\sqrt{\hat{q}^{*}} y\right) \ln \left(2 \cosh \left(\sqrt{\hat{q}^{*}} y\right)\right)}{\int \Delta y \cosh ^{r}\left(\sqrt{\hat{q}^{*}} y\right)}- \\
\alpha K \frac{\int \Delta x H^{r}\left(\sqrt{\frac{q^{*}}{1-q^{*}}} x\right) \ln H\left(\sqrt{\frac{q^{*}}{1-q^{*}}} x\right)}{\int \Delta x H^{r}\left(\sqrt{\frac{q^{*}}{1-q^{*}}} x\right)},
\end{gathered}
$$

and

$$
\begin{aligned}
\mathcal{N}[w(r)]= & \frac{r^{2}}{2} q^{*} \hat{q}^{*}+\ln \left[\int \Delta y 2^{r} \cosh ^{r}\left(\sqrt{\hat{q}^{*}} y\right)\right]- \\
& r \frac{\int \Delta y \cosh ^{r}\left(\sqrt{\hat{q}^{*}} y\right) \ln \left(2 \cosh \left(\sqrt{\hat{q}^{*}} y\right)\right)}{\int \Delta y \cosh ^{r}\left(\sqrt{\hat{q}^{*}} y\right)}+\alpha(K-1) \ln 2+ \\
& \alpha K \ln \left[\int \Delta x H^{r}\left(\sqrt{\frac{q^{*}}{1-q^{*}}} x\right)\right]-\alpha K r \frac{\int \Delta x H^{r}\left(\sqrt{\frac{q^{*}}{1-q^{*}}} x\right) \ln H\left(\sqrt{\frac{q^{*}}{1-q^{*}}} x\right)}{\int \Delta x H^{r}\left(\sqrt{\frac{q^{*}}{1-q^{*}}} x\right)} .
\end{aligned}
$$

The number $\mathcal{N}_{D}$ of domains composing $V_{G}$ is given by $\mathcal{N}[w(r=1)]=-g(1)+w(1)$ :

$$
\begin{aligned}
\mathcal{N}[w(r=1)] & =\frac{\hat{q}^{*}}{2}\left(q^{*}+1\right)-\frac{\int \Delta y \cosh \left(\sqrt{\hat{q}^{*}} y\right) \ln \left(2 \cosh \left(\sqrt{\hat{q}^{*}} y\right)\right)}{\int \Delta y \cosh \left(\sqrt{\hat{q}^{*}} y\right)}+ \\
& +(1-\alpha) \ln 2-2 \alpha K \int \Delta x H\left(\sqrt{\frac{q^{*}}{1-q^{*}}} x\right) \ln H\left(\sqrt{\frac{q^{*}}{1-q^{*}}} x\right)
\end{aligned}
$$

The number $\mathcal{N}_{R}$ of the most numerous domains, i.e. the total number of implementable internal representations, is given by the limit $r=0$. We find

$$
w(r=0)=\frac{1}{2} \hat{q}^{*}\left(1-q^{*}\right)-\int \Delta y \ln \left(2 \cosh \left(\sqrt{\hat{q}^{*}} y\right)\right)-\alpha K \int \Delta x \ln H\left(\sqrt{\frac{q^{*}}{1-q^{*}}} x\right)
$$

and

$$
\mathcal{N}[w(r=0)]=\alpha(k-1) \ln 2+\alpha K \ln \left[\frac{1}{2}+\lim _{r \rightarrow 0} \int_{0}^{\infty} \frac{d x}{\sqrt{2 \pi}} e^{-x^{2} \frac{\left(1-q^{*}+r q^{*}\right)}{2\left(1-q^{*}\right)}}\right] .
$$

The second term of the r.h.s. of above expression is different from zero only if $\lim _{r \rightarrow 0} \frac{r}{1-q^{*}}=$ const., as it happens in the continuous case [2]. In both the continuous and binary cases, beyond a certain value $\alpha_{R}$ of $\alpha$, the number of internal representations which can be realized 
becomes smaller than $2^{(K-1) P}$ as the domains progressively disappear. However, in the binary case the parameters $q^{*}$ does not vanish continuously and a first order RSB transition to a theory described by two order parameters $q_{1}^{*}, q_{0}^{*}$ is required.

At the point where the $w(r)$ vanishes the RS Ansatz must be changed. Following the same RSB scheme as in [2], the one step RSB expression is obtained by breaking the symmetry within each elementary volume and introducing the corresponding order parameters $\left(q_{0}^{*}, \hat{q}_{0}^{*}, q_{1}^{*}, \hat{q}_{1}^{*}, m\right)$ in place of $\left(q^{*}, \hat{q}^{*}\right)$. The free energy reads

$$
\begin{aligned}
g_{R S B}\left(q_{0}=\right. & \left.0, \hat{q}_{0}=0, q_{0}^{*}, \hat{q}_{0}^{*}, q_{1}^{*}, \hat{q}_{1}^{*}, r, m\right)=\frac{1}{2}\left(q_{1}^{*} \hat{q}_{1}^{*}(m-1)+\hat{q}_{1}^{*}+q_{0}^{*} \hat{q}_{0}^{*}(r-m)\right)- \\
& \frac{1}{r} \ln \int \Delta y\left[\int \Delta z\left(2^{m} \cosh ^{m}\left(\sqrt{\hat{q}_{0}^{*}} y+\sqrt{\hat{q}_{1}^{*}-\hat{q}_{0}^{*}} z\right)\right]^{\frac{r}{m}}-\right. \\
& \frac{\alpha}{r}(K-1) \ln 2-\frac{\alpha K}{r} \ln \int \Delta y\left[\int \Delta z H^{m}\left(\frac{\left.\sqrt{q_{1}^{*}-q_{0}^{*}} z+\sqrt{q_{0}^{*}} y\right)}{\sqrt{1-q_{1}^{*}}}\right)\right]^{\frac{r}{m}} .
\end{aligned}
$$

As for the binary perceptron, posing $q_{1}^{*}=1$ leads $\hat{q}_{1}^{*}=\infty$ and

$$
\begin{aligned}
& \left.g_{R S B}\left(q_{0}=0, \hat{q}_{0}=0, q_{0}^{*}, \hat{q}_{0}^{*}, q_{1}^{*}=1, \hat{q}_{1}^{*}=\infty, m, r\right)=\frac{1}{2}\left(q_{0}^{*} \hat{q}_{0}^{*}(r-m)\right)+q_{0}^{*} m\right)- \\
& \frac{1}{r} \ln \int \Delta y 2^{\frac{r}{m}} \cosh ^{\frac{r}{m}}\left(\sqrt{\hat{q}_{0}^{*}} y m\right)-\frac{\alpha}{r}(K-1) \ln 2-\frac{\alpha K}{r} \ln \int \Delta y H^{\frac{r}{m}}\left(\frac{\sqrt{q_{0}^{*}} y}{\sqrt{1-q_{0}^{*}}}\right) .
\end{aligned}
$$

Therefore, we may also write

$$
g_{R S B}\left(q_{0}^{*}, \hat{q}_{0}^{*}, q_{1}^{*}=1, \hat{q}_{1}^{*}=\infty, m, r\right)=\frac{1}{m} g_{R S}\left(\hat{q}^{*}=\hat{q}_{0}^{*} m^{2}, q^{*}=q_{0}^{*}, r^{\prime}=r / m\right)
$$

The saddle point equation with respect to $m$ reads

$$
\frac{\partial g_{R S B}}{\partial m}=-\frac{1}{m^{2}}\left(g_{R S}+r^{\prime} \frac{\partial g_{R S}}{\partial r^{\prime}}\right)=0 .
$$

Such equation is nothing but the condition

$$
w^{R S}\left(\hat{q}^{*}=\hat{q}_{0}^{*} m^{2}, q^{*}=q_{0}^{*}, r^{\prime}=r / m\right)=0,
$$

that, in order to be satisfied, requires

$$
\begin{aligned}
& \hat{q}_{0}^{*}=\frac{\hat{q}_{c}^{*}}{m^{2}}, \\
& q_{0}^{*}=q_{c}^{*}, \\
& m=\frac{r}{r_{c}},
\end{aligned}
$$


where the parameters values $\hat{q}_{c}^{*}, q_{c}^{*}$ and $r_{c}$ are computed at the $w=0$ transition point. From the relations

$$
\frac{\partial r g_{R S B}}{\partial r}=\frac{1}{m} \frac{\partial}{\partial r^{\prime}} r^{\prime} g_{R S} ; \quad r^{2} \frac{\partial g_{R S B}}{\partial r}=r^{\prime 2} \frac{\partial g_{R S}}{\partial r^{\prime}}
$$

it follows

$$
w^{R S B}(r)=\frac{1}{m} w^{R S}\left(r_{c}\right)=0, \mathcal{N}^{R S B}\left[w^{R S B}(r)\right]=\mathcal{N}^{R S}\left[w^{R S}\left(r_{c}\right)\right] .
$$

In Fig.1 we show the behaviour of $r g(r)$ versus $r$ for $\alpha=0.33$. The part of the curve with positive slope cannot exists and hence beyond the $r_{c}$ value the function remains constant and equal to $\mathcal{N}^{R S}\left[w^{R S}\left(r_{c}\right)\right]$.

Just like in the binary perceptron [12] or in the Random Energy Model [13] (for which the one step RSB solution is exact), below $r_{c}$ and for fixed $\alpha$, the system is completely frozen. The function $r g(r)$ behaves like the free energy of the above mentioned systems though in such cases the freezing takes place with respect to the temperature and beyond the critical temperature the free energy is equal to the constant value of the internal energy. The detailed phase diagram in the $\alpha, r$ plane is reported in Fig.2 .

The behaviour of $\mathcal{N}[w(r)]$ versus $w(r)$ for $K=3$ and four different values of $\alpha$ are shown in Fig.3 .

One may observe four different phases:

1. For $\alpha<\alpha_{1}=0.17$, the curve does not touch the $w=0$ abscissa and the domains have volumes between the two values $w_{1}, w_{2}$ for which the ordinate vanishes. For $r<r\left(\mathcal{N}\left[w_{2}\right]=0\right)$ or $r>r\left(\mathcal{N}\left[w_{1}\right]=0\right)$ the RS solution leads to a number of domains less then one and must be rejected. The freezing process takes place at the level of domains in that there are no domains with $w$ values greater then $w_{2}$ and lower then $w_{1}$. The RSB Ansatz substitutes the $q_{0}$ order parameter with $q_{1}, q_{0}$

2. For $\alpha \geq 0.17$, the curve starts at $w=0$ with slope $r_{c}(\alpha)$; hence $\mathcal{N}[w(r)]=$ $\mathcal{N}\left[w\left(r_{c}(\alpha)\right)\right], \forall r<r_{c}(\alpha)$. 
3. At $\alpha=\frac{0.83}{3}=0.277$ we have $r_{c}(\alpha)=0$. The value $\alpha=0.277$, where the zero temperature entropy vanishes, is simply the critical capacity of a binary perceptron with $N / 3$ input units (the size of most numerous domains corresponds to the solution volume of a subperceptron). Beyond this $\alpha$ value, the curve will be enclosed in the region of positive slope $(r \geq 0)$ and the number of internal representations $\mathcal{N}_{R}$ it is no longer $2 \alpha \ln 2$ (i.e. the maximal one) but is given by the value of $\mathcal{N}[w(r)]$ at the starting point of the curve:

$$
\mathcal{N}_{R}=\mathcal{N}\left[w\left(r_{c}(\alpha)\right)\right]
$$

4. At $\alpha=0.41$ the starting slope is $r_{c}(0.41)=1$ and $\mathcal{N}[w(r)=0]=(1-\alpha) \ln 2$ (consistent with the condition $g(1)=(1-\alpha) \ln 2)$.

5. For $\alpha>0.41$, the point $\mathcal{N}[w(r)]=(1-\alpha) \ln 2$ is off the curve and $r_{s}(\alpha)$ is the point at which the two solutions of the saddle point equations lead to the same free energy value, i.e. such that

$$
-\frac{\mathcal{N}\left[w\left(r_{s}(\alpha)\right]\right.}{r_{s}(\alpha)}+w\left(r_{s}(\alpha)\right)=-\frac{1}{r_{s}(\alpha)}(1-\alpha) \ln 2
$$

The starting point of the curve $\left(r_{s}(\alpha)\right)$ grows with $\alpha$. For $r<r_{s}(\alpha)$, the correct saddle point solution is the one giving $\mathcal{N}[w(r)]=(1-\alpha) \ln 2$ independently on $r$, i.e. the isolated point marked in Fig.3 . The switch between the two solutions can be understood by noticing that it correspond to the only possible way of obtaining $g(1)=(1-\alpha) \ln 2$ for $\alpha<0.41$. Moreover, its physical meaning is that for $r<r_{s}(\alpha)$ it is not necessary to distinguish among different domains in that $V_{G}$ is dominated by the domains of zero entropy independently on the freezing process.

6. For $\alpha=0.56$ only one point remains.

7. At $\alpha=1$ also the point disappears.

In the following section we will compare the behaviour of $\mathcal{N}_{R}$ and $\mathcal{N}_{D}$ computed for $K=3$ with the results of numerical simulations on finite systems. 
Very schematically we have

$$
\mathcal{N}_{R}=\left\{\begin{array}{lc}
2 \alpha(\ln 2) & \alpha \leq 0.277 \\
\mathcal{N}\left[w\left(r_{c}(\alpha)\right)\right] & 0.277<\alpha<0.41 \\
(1-\alpha) \ln 2 & \alpha>0.41
\end{array}\right.
$$

and

$$
\mathcal{N}_{D}= \begin{cases}\mathcal{N}[w(r)=1] & \alpha \leq 0.41 \\ (1-\alpha) \ln 2 & \alpha \geq 0.41\end{cases}
$$

The overall scenario arising from the analytical computation may be summarized briefly as follows. We find a freezing transition at $\alpha_{2}=0.41$ within the domains. For values of $\alpha>\alpha_{2}$ the domains, though still distributed over the whole space of solution $\left(q_{0}=0\right)$, are composed by configurations with overlap $q^{*}=1$. The point $\mathcal{N}_{D}=0$ is the symmetry breaking point also corresponding to the critical capacity of the model $\alpha_{c}=1$ [5].

\section{NUMERICAL SIMULATIONS}

We have checked the above scenario by performing two distinct sets of extended numerical simulations on the weight space structure of a parity machine with binary weights and three hidden units.

In the first simulation we have measured both the dimension $w(r)$ and the number $\mathcal{N}[w(r)]$ of domains depending on the loading parameter $\alpha$. In particular we have considered the cases $r=1$ and $r=0$ giving respectively the measure of the number $\mathcal{N}_{D}$ of domains contributing to the total Gardner volume $V_{G}$ and the overall number $\mathcal{N}_{R}$ of implementable internal representation. In the second set of simulations we have reconstructed the plot of $r g(r)$ and $\mathcal{N}[w(r)]$ as function of $r$ and for fixed $\alpha$.

The numerical method adopted is the exact enumeration of the configurations $\left\{J_{\ell i}\right\}$ on finite systems. Very schematically the procedure is the following.

1. choose $P$ random patterns; 
2. divide, for every subperceptron, the set of $2^{n}(n=N / 3)$ configurations in subsets labeled by the vectors $\overrightarrow{\tau_{\ell}}\left(\tau_{\ell}^{\mu}=\operatorname{sign}\left(\vec{J}_{\ell} \cdot \xi_{\ell}^{\mu}\right)\right) \ell=1,2,3$

3. try all the subsets combinations between the three subperceptron and identify the domains of solutions as those which satisfy $\prod_{\ell} \tau_{\ell}^{\mu}=1, \forall \mu$.

The above scheme yields a parallel enumeration and classification of the $2^{n}$ weights configurations in the three subperceptron. To avoid ambiguities in the signs of the hidden fields the number of inputs connected to each hidden unit must be odd. The sizes of the systems taken under consideration are $N=15,21,27$ for the first type of simulation and $N=15,21,27,33$ for the second.

More in detail, the three steps of the numerical procedure are the following.

1. We use Gaussian patterns in order to reduce finite sizes effects (as has been done for the binary perceptron [3, 12, 15, 14]). From the replica method one expects that the results are equivalent to those of binary weights in that they depend only on the first two moments of the quenched variables.

2. The classification of the $2^{n}$ weights configurations is as follows: we start with $\vec{J}=(-1,-1, \ldots,-1)$. Next we compute for every $\ell$ and $\mu$ the field $a_{\ell}^{\mu}=-\sum \xi_{\ell i}^{\mu}$ together with its $\operatorname{sign}\left(\tau_{\ell}^{\mu}\right)$ so that the vector $\vec{\tau}_{\ell}$ labels the first subset. The subsequent $J$ configurations are generated by means of the Gray code which flips just one of the $J_{i}$ components at each time step and allows to update the field values with a single operation $a_{\ell}^{\mu}=a_{\ell}^{\mu}+2 \xi_{\ell i}^{\mu}$ (this reduces the number of operations by a factor $n)$. Then, depending on whether the vector $\overrightarrow{\tau_{\ell}}$ is different from the previous one or not, we use $\overrightarrow{\tau_{\ell}}$ as new label of the second subset or increment the number of vectors contained in the first one. We thus proceed in this way to scan the $\vec{J}$ configurations. If $P$ varies from 1 to $3 n$, every $\vec{J}$ configuration is classified $n$ times on each subperceptron. At the end we obtain 3 ( $P$ fixed) or $3 n$ ( $\mathrm{P}$ varying from 1 to $3 \mathrm{n}$ ) tables whose columns (in number $\leq 2^{n}$ ) are the $\overrightarrow{\tau_{\ell}}$ vectors 
labeling the subsets and to which are associated the numbers of $J_{\ell}$ belonging to each subset.

3. Finally, in the case of a given $P$, we take a column in each of the three tables and verify whether the product between the two chosen columns from the first two tables is equal to the column of the third one. If so, the internal representation given by the three columns matrix is implementable and the volume of the corresponding domain is the product of the numbers of $J_{\ell}$ belonging to the subset.

Once the domains volumes $\left(V_{\mathcal{T}}\right)$ have been measured, we compute:

$$
\begin{gathered}
-r g(r)=\ln \sum_{\mathcal{T}} V_{\mathcal{T}}^{r}, \\
-w(r)=\frac{\sum_{\mathcal{T}} V_{\mathcal{T}}^{r} \ln V_{\mathcal{T}}}{\sum_{\mathcal{T}} V_{\mathcal{T}}^{r}},
\end{gathered}
$$

(which is the domain size computed on the saddle point of the partition function) and

$$
\mathcal{N}[w(r)]=-r g(r)+r w(r) .
$$

For the first set of simulations, the above functions are computed just for $r=0,1$ and the averages are taken over $10000(\mathrm{~N}=15), 1000(\mathrm{~N}=21)$ or $50(\mathrm{~N}=27)$ samples. In the case of the second set of simulations, in order to allow for a comparison between all the finite sizes considered, $\alpha$ is settled at $\alpha=0.33 . r$ runs from -1.5 to 3 and the average is done over $10000(\mathrm{~N}=15, \mathrm{~N}=21), 5000(\mathrm{~N}=27)$ or $200(\mathrm{~N}=33)$ samples. The statistical errors bars are within $0.1 \%$.

As shown in Fig.4, both theoretical and experimental results give $g(r=1)=-(1-\alpha) \ln 2$ which coincides with the annealed approximation (so that the total volume is reduced simply to a half for every added pattern and $\alpha_{c}=1$ 《4). At the value $\alpha=0.277$ (Fig. 5), the total number of internal state vectors belonging to the most numerous volumes (i.e. volumes characterized by $r=0)$ becomes non-extensive $(w(r=0)=0)$. Beyond such a value and in 
perfect agreement with simulations, the correct solution is given by one step of RSB which, in fact, predicts $w(r=0)=0, \forall \alpha>0.277$.

As shown in Fig.6, beyond $\alpha=0.27$ the domains begin to disappear and the number of internal representations ceases to be constant (equal to $2 \alpha \ln 2$ ) and starts to decrease with $\alpha$. For $r=1$ the freezing transition takes place at $\alpha=0.41$, see Fig.7 and Fig.8 .

As shown in Fig.1, for $\alpha=0.33$ the theoretical value for the freezing transition is $r_{c}=0.4$; for $r<r_{c}$ the slope of the curve $r g(r)$ is zero (it cannot become positive) and $r g(r)=-\mathcal{N}\left[w\left(r_{c}\right)\right]=-0.43$. Finally, the plot of $\mathcal{N}[w(r)]$ versus $w(r)$, for $\alpha=0.33$, is given in Fig.9.

\section{CONCLUSION}

In this paper we have applied the internal representation volumes approach to the case of binary multilayer networks, in particular to the non-overlapping parity machine. The chief result of our study consists in a detailed comparison between the analytical prediction and the numerical simulations, allowing for a definitive confirmation of the method. The detailed geometrical structure of the weights space predicted by the theory, both $\mathcal{N}_{D^{-}} \mathcal{N}_{R}$ as well as the RSB transitions within the volumes, turn out to be in remarkable agreement with the numerical simulations performed on finite systems.

As a general remark, let us emphasize that multilayer neural networks with binary weights behave differently from their continuous counterpart. While the breaking of symmetry in the former occurs inside the representations volumes, we have already shown that in the case of real valued couplings the transition takes place between different volumes [2]. Therefore, the richness of the distribution of internal representations found in the continuous case, i.e. the presence of a "finite" number of macroscopic regions in the weight space containing a very large number of different internal representations, is partially lost when one deals with discrete weights.

The method can be easily extended [2,[1] to address the rule inference capability problem. 
Thus, another very interesting and important issue related to the present approach would be the study of the distribution of metastable states arising from a gradient learning process. Work is in progress along these lines. 


\section{REFERENCES}

*Email: cocco@roma1.infn.it

† Email: monasson@physique.ens.fr ; LPTENS is a unité propre du CNRS, associée à l'Ecole Normale Supérieure et à l'Université de Paris-Sud.

\# Email: zecchina@to.infn.it ; work supported by an Elsag-ISI grant.

[1] J. Hertz, A. Krogh, R. G. Palmer, Introduction to the Theory of Neural Computation, Addison-Wesley Pub. (1991)

[2] R. Monasson, R. Zecchina, Phys. Rev. Lett 75, 2432 (1995)

[3] E. Gardner, J. Phys. A 21, 257 (1988)

E. Gardner, B. Derrida, J. Phys. A 21, 271 (1988)

[4] E. Barkai, D. Hansel, I. Kanter, Phys. Rev. Lett. 65, 2312 (1990)

E. Barkai, D. Hansel, H. Sompolinsky, Phys. Rev. A 45, 4146 (1992)

A. Engel, H.M. Kohler, F. Tschepke, H. Vollmayr, A. Zippelius, Phys. Rev. A 45, 7590 $(1992)$

[5] E. Barkai, I. Kanter, Europhys. Lett. 14, 107 (1991)

[6] M. Opper, Phys. Rev. E 51, 3613 (1995)

[7] Correlation of internal representations in feed-forward neural networks, preprint condmat/9604070 (1996)

[8] G.J. Mitchison, R.M. Durbin, Bio. Cybern. 60, 345 (1989)

[9] M. Mezard, G. Parisi, M.A. Virasoro, Spin Glass Theory and Beyond, World Scientific, Singapore, (1987)

[10] R. Monasson, D. O’Kane, Europhys. Lett. 27, 85 (1994)

[11] R. Monasson, R. Zecchina, Learning and Generalization Theories of Large Committee- 
Machines, to be published in Int. J. Mod. Phys. B

[12] W. Krauth, M. Mézard J. Physique, 50, 3057 (1989)

[13] B. Derrida, Phys. Rev. B24, 2613 (1981)

[14] W. Krauth, M. Opper, J. Phys. A 22, L519 (1989)

[15] H. Gutfreund, Y. Stein, J. Phys. A 23, 2613 (1990) 
FIG. 1. $r g(r)$ versus $r$ for $\alpha=0.33$ and $K=3$. The theoretical curve corresponds to the continuous lines whereas the marked curves are the numerical results obtained for $N=15,21,27,33$.

FIG. 2. Freezing transition for the binary parity machine for $K=3$. The $r_{c}(\alpha)$ line separates the RS and the RSB phases. The three marked points describe the transition at $\alpha=$ const. and correspond to the following values of the parameters and the entropy: (a) $q^{*}(r), \hat{q}^{*}(r), \mathcal{N}[w(r)]$, (b) $q^{*}\left(r_{c}\right), \hat{q}^{*}\left(r_{c}\right), \mathcal{N}\left[w\left(r_{c}\right)\right]$ and (c) $\hat{q}_{0}^{*}=\hat{q}^{*}\left(r_{c}\right) / m^{2}, q_{0}^{*}=q^{*}\left(r_{c}\right), q_{1}^{*}=1, \hat{q}_{1}^{*}=\infty, m=r / r_{c}$, $\mathcal{N}[w(r)]=\mathcal{N}\left[w\left(r_{c}\right)\right]$.

FIG. 3. $\mathcal{N}[w(r)] / \alpha$ versus $w(r)$ for $\alpha=0.177,0.277,0.41,0.495$. The dotted points signal the starting points $\left(r_{c}\right)$ corresponding to $w\left(r_{c}\right)=0$ and the points with slope $r=0$ and $r=1$. Notice that the diamond bolded point belongs to the dashed-dotted curve.

FIG. 4. $g(r=1)$ versus $\alpha$. The theoretical curve (continuous line) is compared with the numerical outcomes (marked points).

FIG. 5. $-w(r=0)$ versus $\alpha$ (theoretical continuous line and numerical points). The $r=0$ freezing transition appears at $\alpha=0.277$.

FIG. 6. $\mathcal{N}_{R} / \alpha$ versus $\alpha$ (theoretical continuous line and numerical points).

FIG. 7. $-w(r=1)$ versus $\alpha$ (theoretical continuous line and numerical points). The $r=1$ freezing transition appears at $\alpha=0.41$.

FIG. 8. $\mathcal{N}_{D} / \alpha$ versus $\alpha$ (theoretical continuous line and numerical points).

FIG. 9. $\mathcal{N}[w(r)] / \alpha$ versus $w(r)$ for fixed $\alpha=0.33$. 
Figure 1

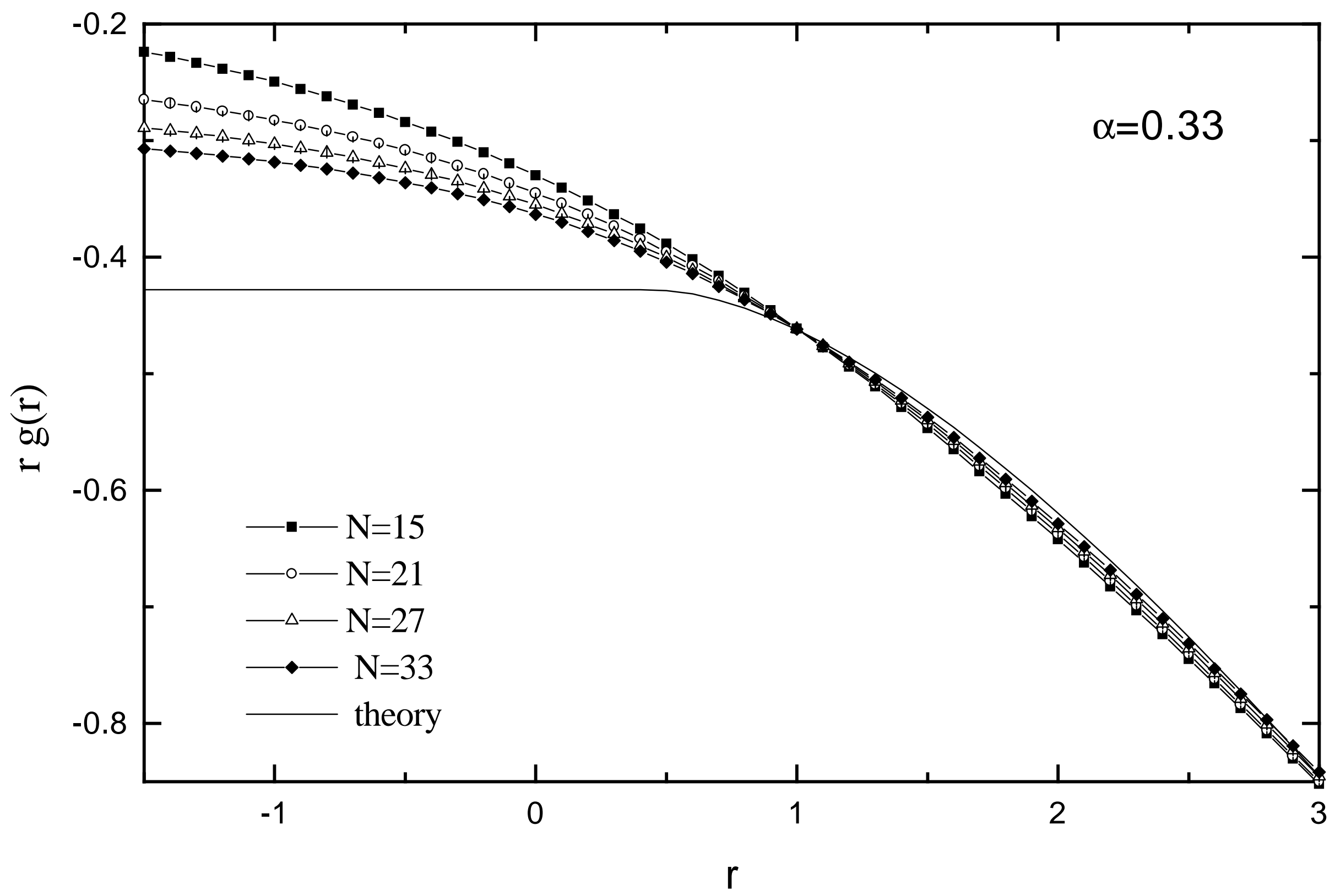


Figure 2

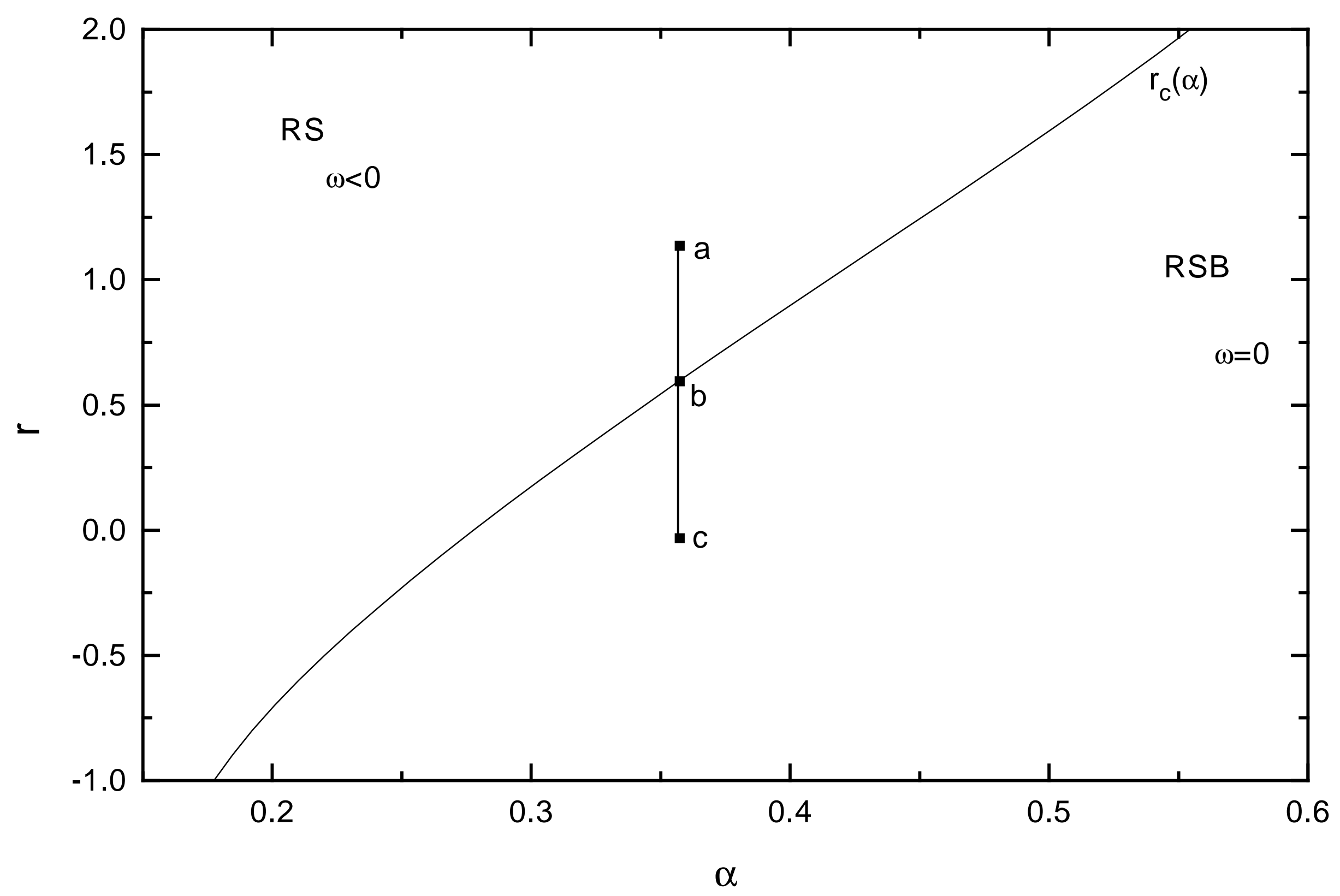


Figure 3

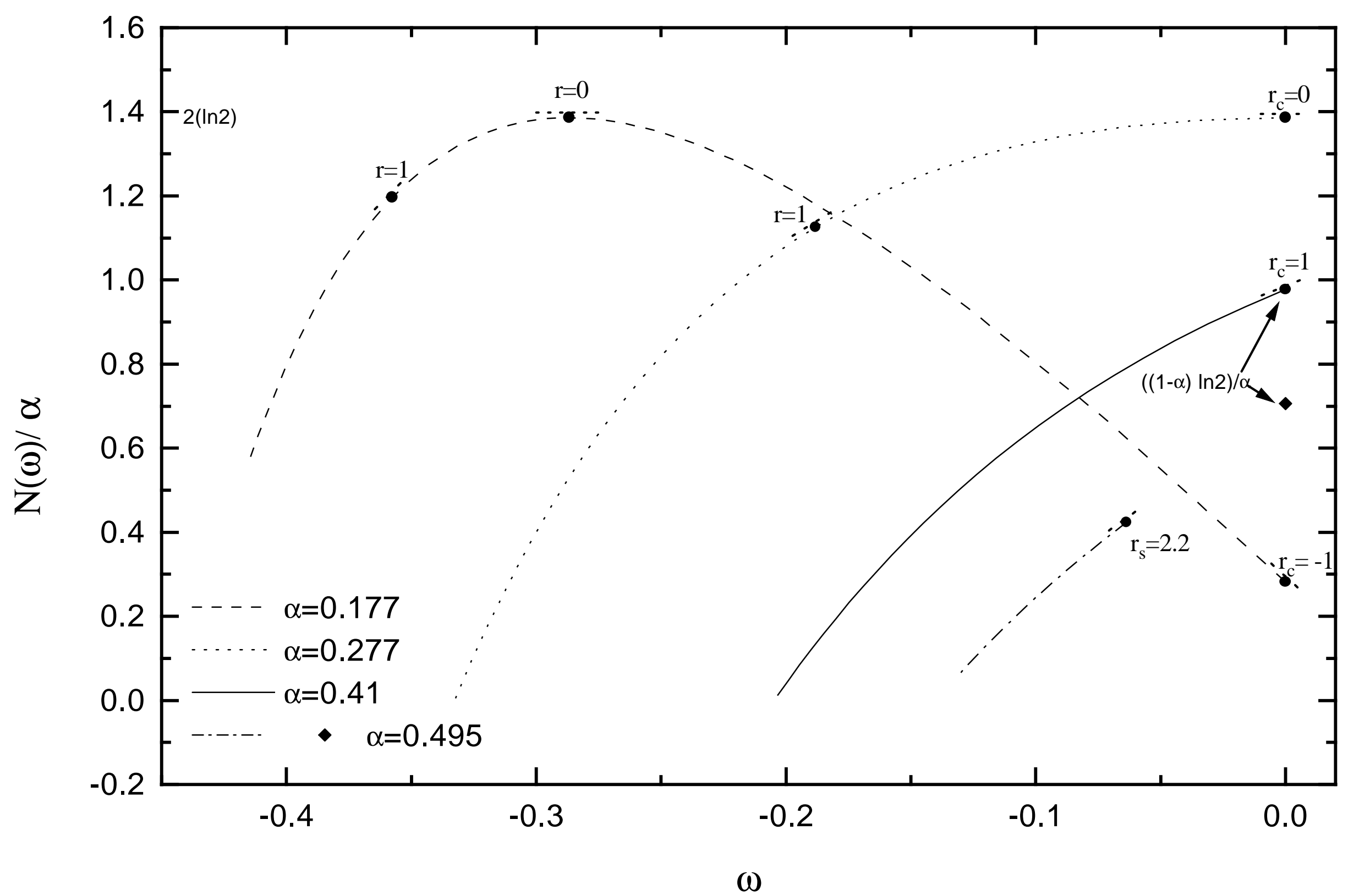


Figure 4

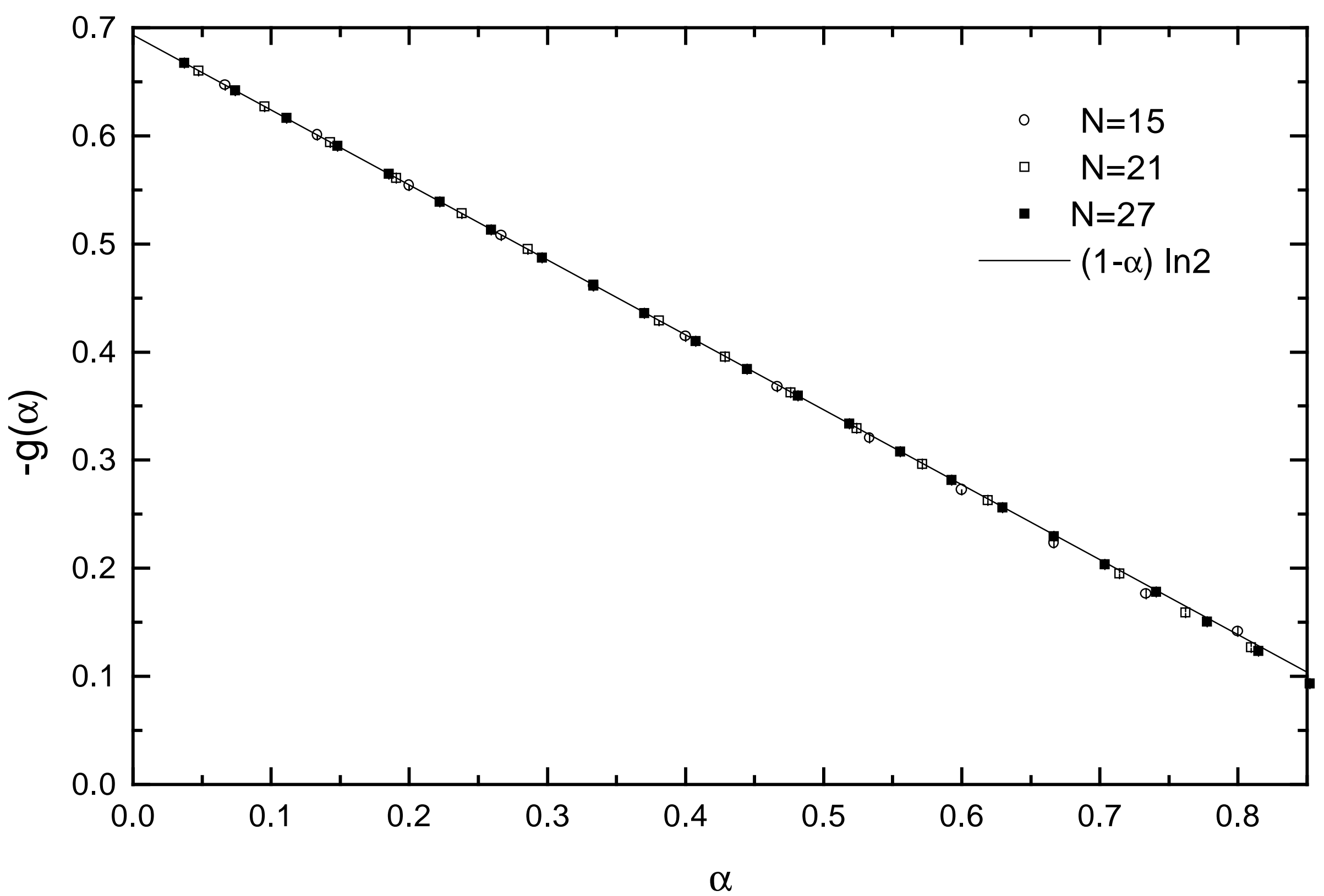


Figure 5

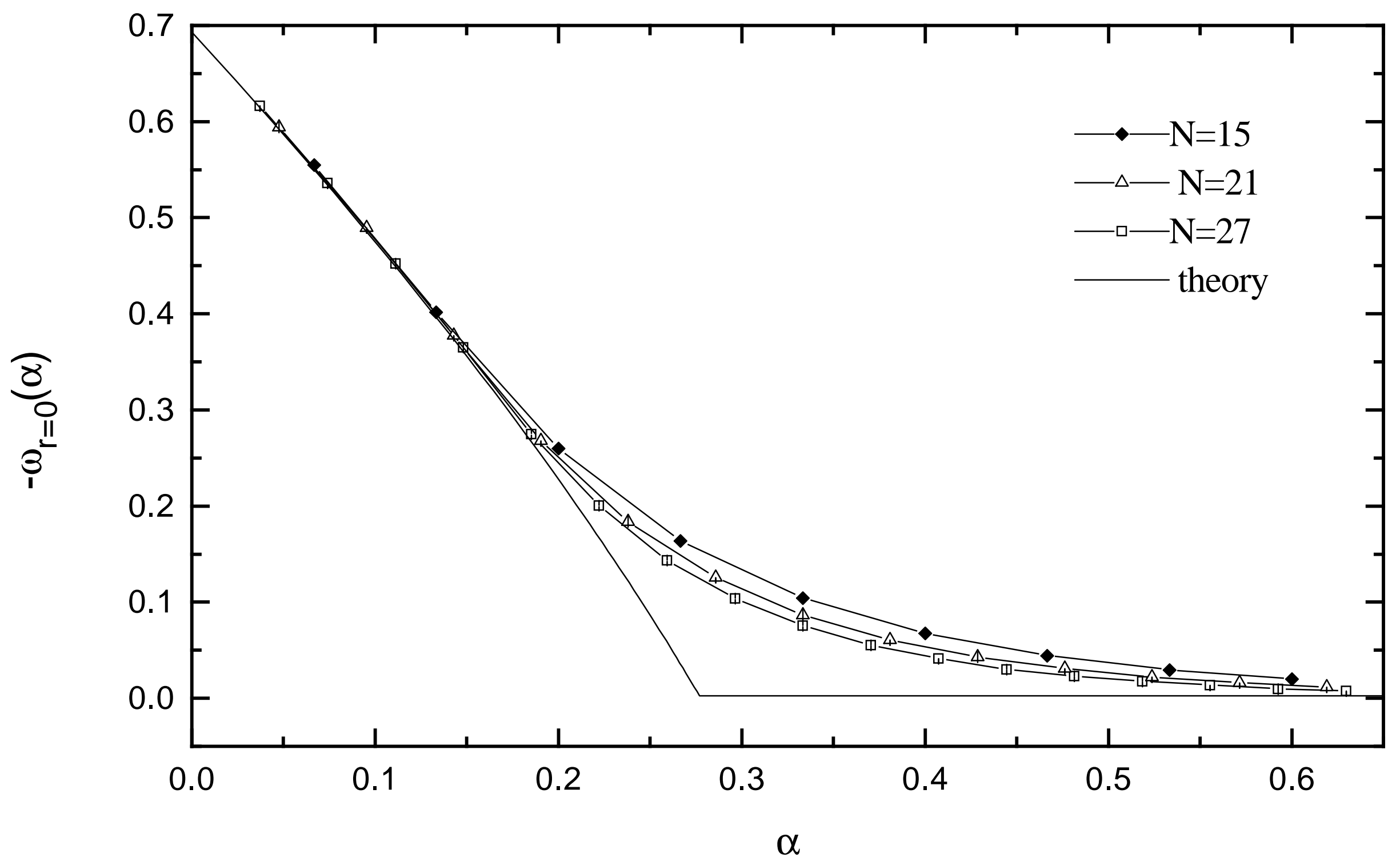


Figure 6

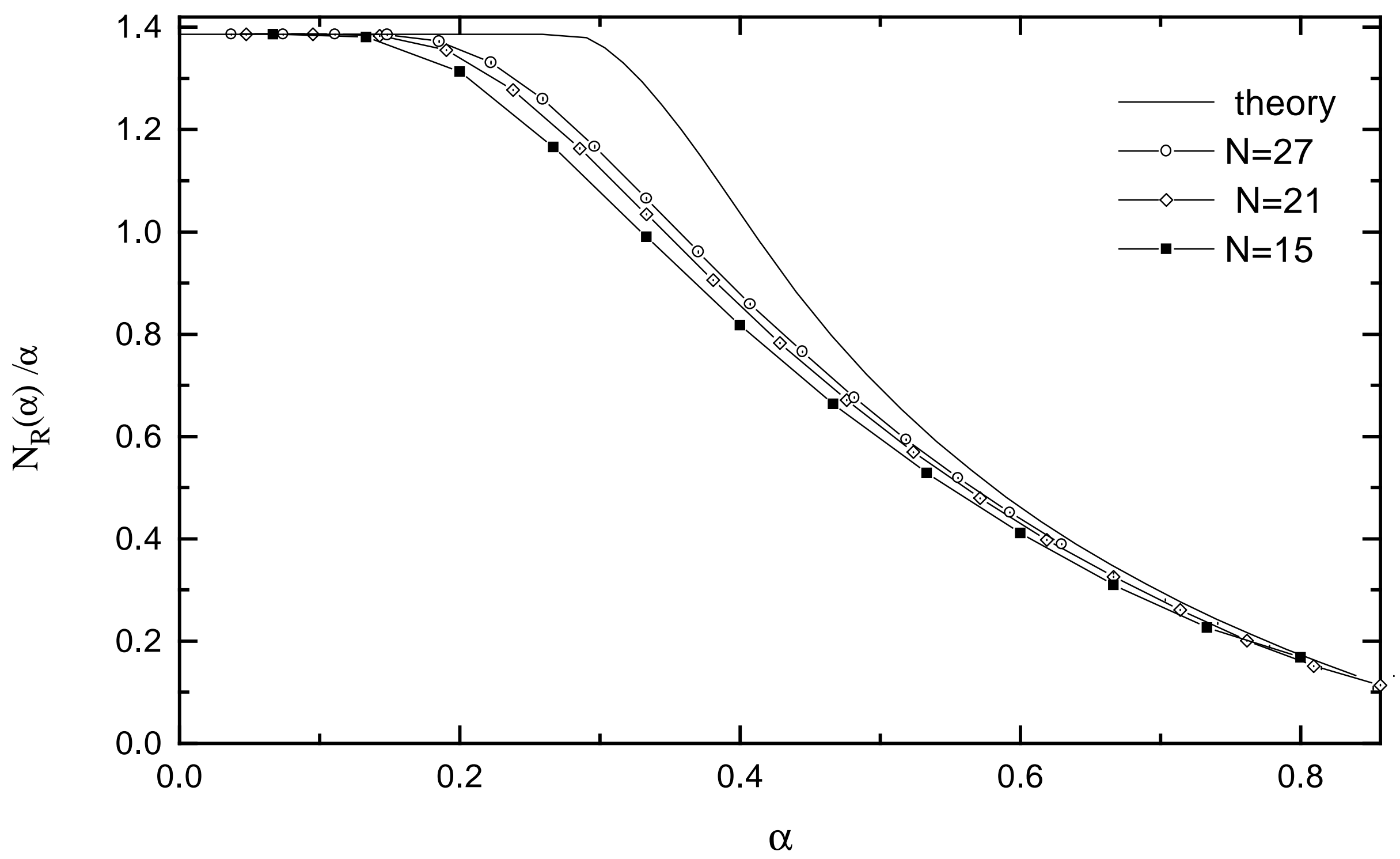


Figure 7

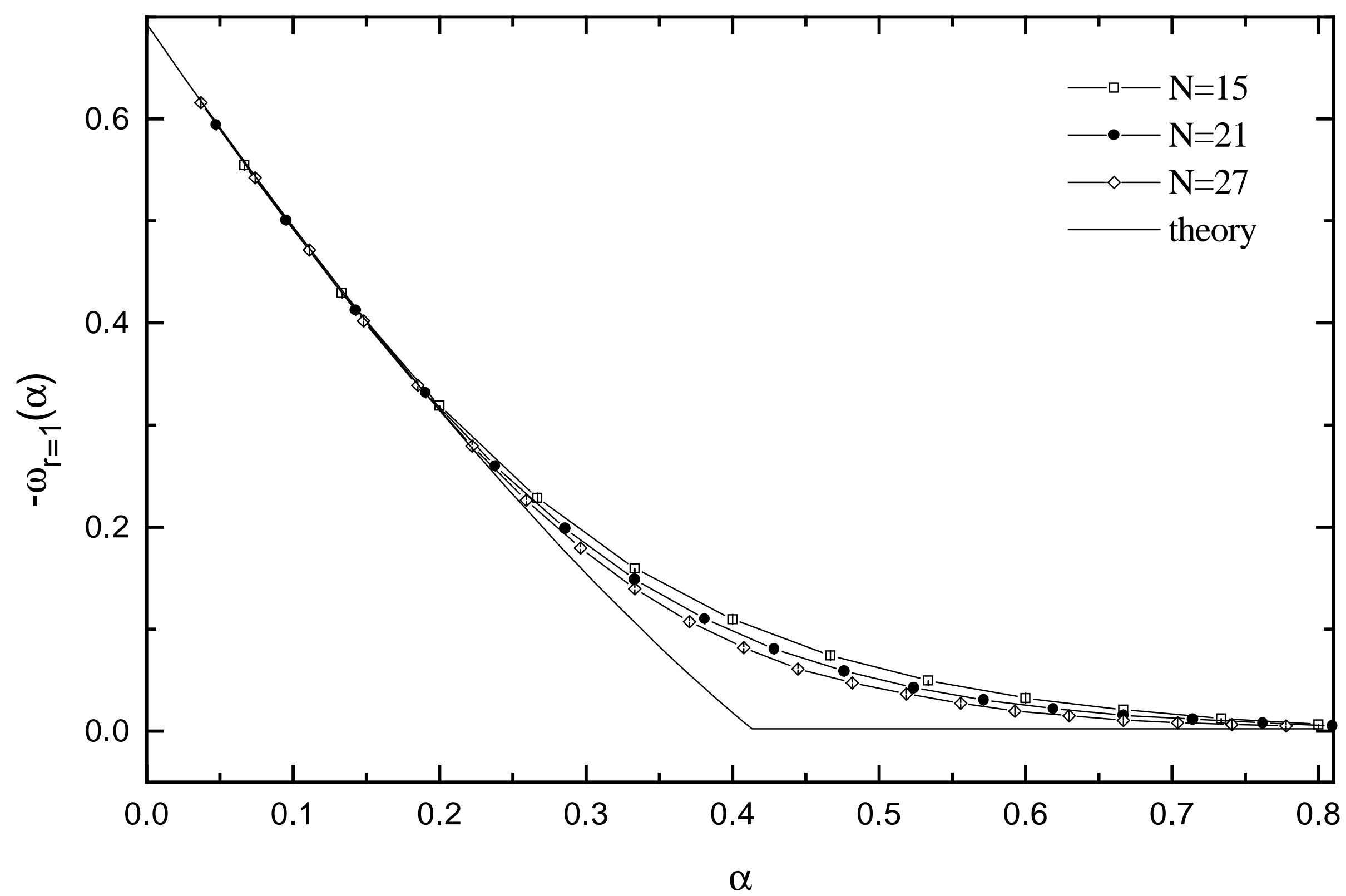


Figure 8

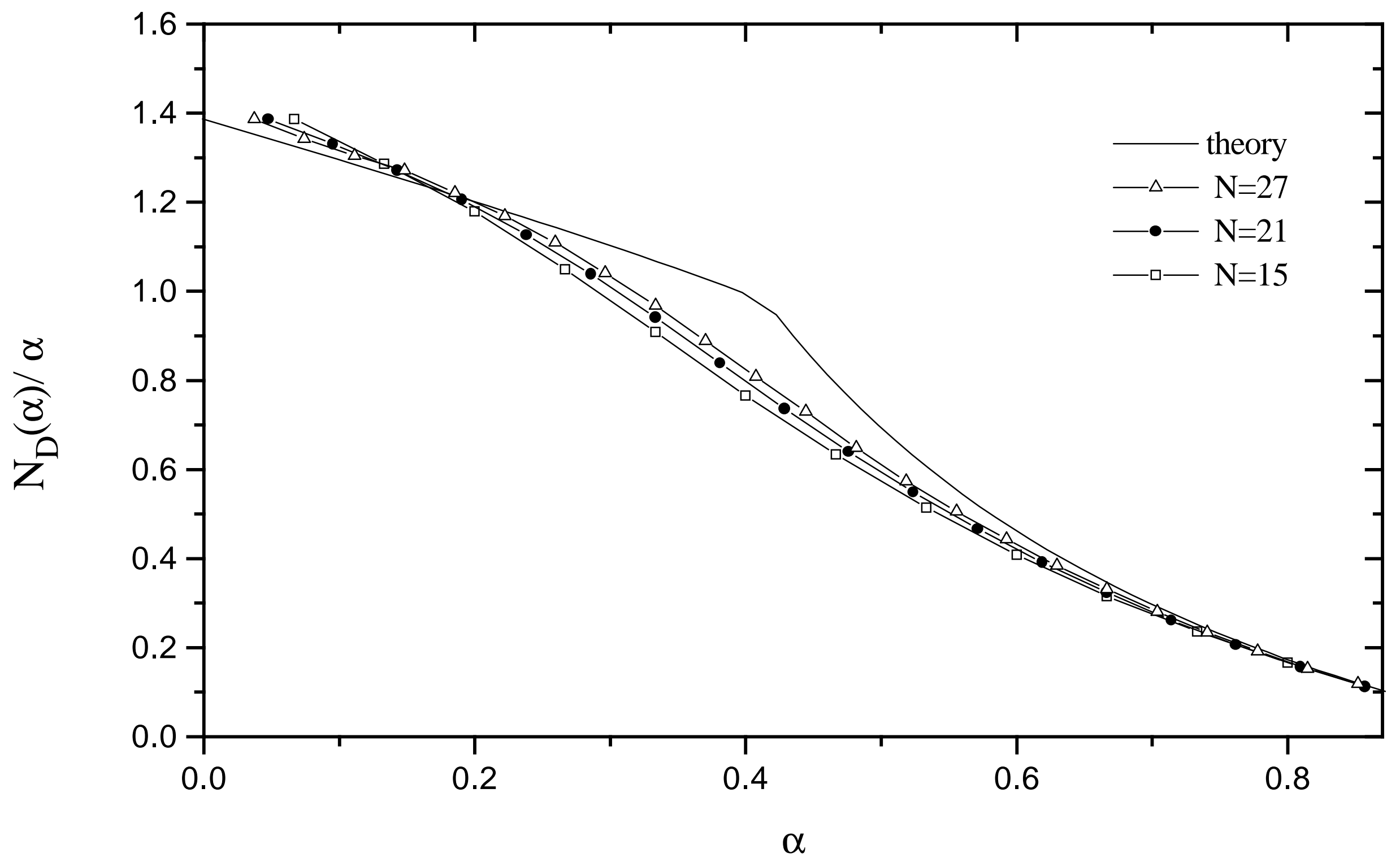


Figure 9

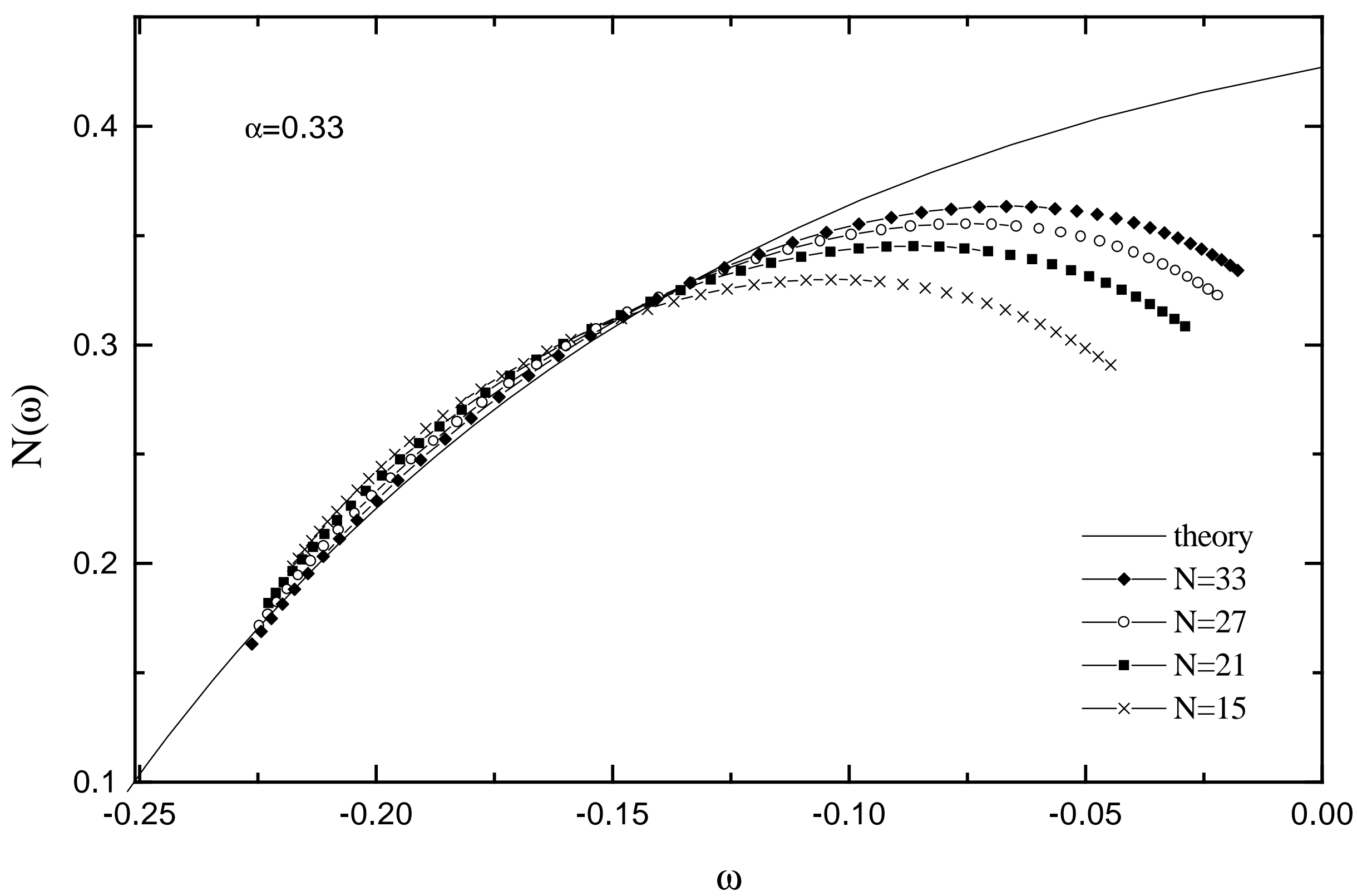

